most comfortable. METHODS/STUDY POPULATION: We have used several strategies to enable investigators to gain input from stakeholders in rural and frontier areas. If the research focuses on rural populations, we hold the Studio at a central location, usually at a restaurant in a private room, if this is available. If the investigator wants to hear from both rural and urban residents, we use videoconferencing via Skype or FaceTime when individuals have enough bandwidth to support it and/or feel comfortable using this technology. For those who have dial-up or no internet access, we provide a conference call line Trusting relationships are essential to creating a safe space in which stakeholders from communities facing health disparities can provide consultations to researchers. When an investigator wishes to consult with stakeholders from one racial/ethnic community, we contract with a leader or trusted member of that community to recruit appropriate stakeholders. The Studio is co-facilitated by a CCET staff member and a community leader in the community's preferred language, with the leader translating for the CCET staff member. For Studios that involve stakeholders from multiple communities and that are conducted in English, we provide translators, if appropriate. Stakeholders using translation may be present in the room with other Studio participants or may be on the phone. RESULTS/ANTICIPATED RESULTS: Of the 35 Studios we have held, five have been held in rural locations and another five have included one or more rural/frontier stakeholders participating via phone or videoconferencing. Six Studios have been co-facilitated with community leaders and four others have included translators. Almost all Studios we have held in English have included individuals representing diverse communities. Anonymous surveys completed at the end of Studios show that participants report the following on 5-point Likert scales:. The facilitator managed the allotted time so that my voice was heard (67\% strongly agree; $33 \%$ agree). The relevant experts were present at the Studio (78\% strongly agree; $22 \%$ agree). I was satisfied with the Studio session (78\% strongly agree; $22 \%$ agree). The Studio process was worth my time (89\% strongly agree; $11 \%$ agree). The feedback provided by the community experts will improve the research project (68\% strongly agree; $44 \%$ agree). Participants were also asked what they felt was their contribution to the research project. Among the most common themes were: increased researcher's understanding of the community, increased researcher's sensitivity to the community, provided feedback on the feasibility of the project, provided ideas on recruiting research participants, provided ideas for how to use the project results to benefit the community, and provided ideas on how to inform the community about the project. All participants said that they would participate in a Studio again. DISCUSSION/SIGNIFICANCE OF IMPACT: Studies at all stages in the research life cycle can be strengthened through consultations with community experts. These stakeholders can inform needs assessments, provide input on study design, supply critical information on supports and barriers to research participation, review study instruments for readability and cultural appropriateness, provide feedback on recruitment and educational materials, and inform dissemination of research results, among others. These consultations provide the most benefit to researchers when they include the voices of as broad a range of stakeholders as possible. We have shown that it is feasible to include stakeholders who live in rural and frontier areas in Studio consultations. We also have developed successful methods for holding Studio consultations with stakeholders who are members of communities facing health disparities and who speak multiple languages. This expanded representation in Community Engagement Studios strengthens research studies.

\section{Addressing Community Health Needs through Community Engagement Research Advisory Boards} Christi Patten, $\mathrm{PhD}^{1}$, Monica L. Albertie, $\mathrm{MHA}^{1}$, Chara A. Chamie, $\mathrm{MPH}^{1}$, Tabetha A. Brockman, $\mathrm{MA}^{1}$, Mary Gorfine ${ }^{1}$, Rosa Nicholas ${ }^{1}$, Martha J. Bock, BA ${ }^{1}$, Janet M. Okamoto, $\mathrm{PhD}^{1}$, Sumedha G. Penheiter, PhD $^{1}$ and Joyce E. Balls-Berry, PhD $^{1}$ ${ }^{1}$ Mayo Clinic

OBJECTIVES/SPECIFIC AIMS: Over $80 \%$ of CTSA programs have a community advisory board (CAB), an effective strategy to increase community engagement (CE) in research. Little is known about how the research discussed with CABs aligns with community priorities (i.e., bi-directionality). This program evaluation assessed the health topics presented by researchers to the CABs linked to our CE Program at all three Mayo Clinic sites (MN, AZ, and FL) for relevance to local community needs. METHODS/STUDY POPULATION: Two coders classified Mayo researcher presentations to our CABs from 2014-2018 for relevance to needs identified in the local 2013 and/or 2016 County Health Needs Assessments and specific topic(s); with high levels of agreement (Kappa=0.90). RESULTS/ANTICIPATED RESULTS: Overall, of the 65 presentations $41(63 \%)$ addressed one or more local health needs $(47 \% \mathrm{MN}$, $60 \% \mathrm{FL}, 80 \% \mathrm{AZ}$ ). Cross-cutting health topics addressed at 2 sites were physical activity/obesity/nutrition and mental health. DISCUSSION/SIGNIFICANCE OF IMPACT: Findings were shared with our CABs to obtain input on future directions. The FL and AZ $\mathrm{CABs}$ are systematic in seeking out or initiating research projects that address local health needs, an approach the MN site is interested in adopting. Ultimately, it is important to demonstrate improved health outcomes with CTSA-based CE research strategies. Understanding community health needs and depth of researchers in those areas may help to focus priorities for demonstrating such outcomes.

3191

\section{Adolescent Substance Use: School and Community Perspectives on School-Based Interventions}

Sycarah Fisher

University of Kentucky Center for Clinical and Translational Science

OBJECTIVES/SPECIFIC AIMS: Fifty percent of adolescents have tried an illicit drug and $70 \%$ have tried alcohol by the end of high school. Further, despite 7-9\% of youth 12-17 meeting criteria for a substance use disorder only 1 in 10 actually receive it. Screening, Brief Intervention, and Referral to Treatment (SBIRT) is an evidence based process that facilitates early identification and treatment for adults and adolescents in community (primary care) facilities. Despite the documented effectiveness of SBIRT, no research has examined the implementation of SBIRT in school settings by schoolbased mental health personnel. The purpose of the present study was to identify facilitators and barriers to SBIRT implementation by school-based personnel in secondary schools. METHODS/STUDY POPULATION: Participants included 30 school and community service providers including: teachers, school counselors, school psychologists, school administrators (principals and central office staff), city council members, school board members, community mental health services providers as well as state level individuals from the department of Adolescent Substance Use and the Office of Drug Control Policy. Interview guides were developed using the 